P3 IMPROVING ERCP TISSUE ACQUISITION IN MALIGNANT STRICTURES WITH TRANSPAPILLARY ENDOBILIARY BIOPSIES

Wafaa Ahmed*, Shwan Karim, Christopher Wadsworth. Imperial College Healthcare NHS Trust, London, UK

\subsection{6/gutjnl-2020-bsgcampus.78}

Introduction Endoscopic retrograde cholangiopancreatography (ERCP) is performed in patients with panctreaticobiliary strictures to allow pathological diagnosis of malignancy and although brush cytology has been shown to have high specificity its sensitivity is low. Endoscopic transpapillary biopsies are obtained alongside brush cytology in our centre. We sought to assess whether this technique increased diagnostic accuracy.

Methods Endoscopy reporting system was retrospectively interrogated for all patient undergoing ERCP where cytology and endobiliary biopsies were obtained from January 2015 to December 2019. Patients with no confirmed diagnosis were excluded. Patient demographics, comorbidities, cytology and histology were recorded. Endobiliary biopsies were taken by advancing paediatric forceps into the bile duct after sphincterotomy under fluoroscopic guidance.

Results A total of 105 ERCPS (Male=68, 64.8\%, Female $=$ $37,35.2 \%)$ were done where both endobiliary cytology and biopsies were taken. Median age was 67.5 years old (range 28.4-90.7). 81 (75.7\%) had at least one co-morbidity. Median number of biopsies taken was 4 (range 1-10) of biopsies taken, 8 did not have no of biopsy's recorded. 67 cases had a malignant stricture confirmed after MDM discussion.

The sensitivity of cytology alone was $34.3 \% \quad(23.2 \%$ to $46.9 \%)$ compared to $56.7 \%$ (44.0\% to $68.8 \%$ ) for histology alone. Combined, their sensitivity was greater than for either alone $64.2 \%$ (51.5\% to $75.5 \%)$. For patients with confirmed cholangiocarcinoma $(\mathrm{N}=23)$, cytology alone had a sensitivity of $43.5 \%(23.2 \%$ to $65.5 \%)$ as compared to $73.9 \%(51.6 \%$ to $89.8 \%$ ) for histology. The combined sensitivity in cholangiocarcinoma was also greater than for either alone $82.6 \%$ $(61.2 \%$ to $95.1 \%)$. There were no false positives (Specificity= $100 \%)$.

There were 4 incidence of post ERCP pancreatitis, none of which met severe criteria and 1 case of a local retroperitoneal perforation at D2/D3 which settled spontaneously.

Conclusions The use of endobiliary biopsies alongside brush cytology in cases of malignant strictures increases sensitivity, particularly in cases of cholangiocarcinoma. Endobiliary biopsy in itself did not increase risk of complication.

\section{P4 CLINICAL AND ENDOSCOPIC CHARACTERISTICS ASSOCIATED WITH INTERVAL UPPER GASTROINTESTINAL CANCERS: A SYSTEMATIC REVIEW AND META-ANALYSIS}

1,2Leo Alexandre*, 'Theo Tsilegeridis-Legeris, 1,2Stephen Lam. 'Norwich Medical School, University of East Anglia, Norwich, UK; ${ }^{2}$ Norfolk and Norwich University Hospital, Norwich, UK

\subsection{6/gutjnl-2020-bsgcampus.79}

Introduction The prognosis of upper gastrointestinal (UGI) cancers generally is very poor and attributed to advanced stage at diagnosis. Approximately one in every twelve patients with an UGI cancer in the UK receives a gastroscopy within three years prior to diagnosis (an interval cancer). This systematic review aimed to (1) determine the clinical and endoscopic characteristics associated with interval UGI cancers (compared with incident cancers), and (2) quantify the proportion of individual endoscopic findings in endoscopies within three years preceding diagnosis of interval cancer.

Methods We searched MEDLINE and Embase from Jan 1, 2000, to Feb 7, 2020, for studies comparing the characteristics of interval and incident UGI cancers, and studies reporting the proportion of findings at the initial 'cancer-negative' endoscopy in those with interval cancer. We synthesised results using random effects meta-analysis. This review is registered on PROSPERO, CRD42019125780.

Results A total of 997 citations were screened and 13 studies were included in the meta-analysis, comprising 44315 UGI malignancies, of which $5067(11.4 \%)$ were interval cancers. In studies reporting the interval between the first 'cancer-negative' endoscopy and diagnosis of cancer (within 3 years), the median interval was 18 months. There were no significant differences in age or gender between interval and incident UGI cancers. Patients with interval cancers were less likely to present with dysphagia (OR 0.38, 95\% CI $0.17-0.88$ ) or weight loss (OR 0.50 , 95\% CI $0.28-$ 0.92). There were no differences in presentation with anaemia (OR 0.62, 95\% CI 0.23-1.64), GI bleeding (OR 1.20, 95\% CI $0.75-1.91$ ), or vomiting (OR 0.69 , 95\% CI $0.36-$ 1.30). Gastro-oesophageal reflux (OR 2.69, 95\% CI 2.283.18) was associated with a higher odds of interval malignancy. Endoscopist experience was not associated with interval cancers (OR 1.16, 95\% CI 0.79-1.69). Intestinal metaplasia (IM) was associated with interval gastric cancer (OR 4.85, 95\% CI 1.86-12.69). For gastric cancer, the most common abnormalities in the first 'cancer-negative' endoscopy prior to diagnosis were IM (41\%), gastritis $(36 \%)$ and gastric atrophy (31\%). For oesophageal cancer, the equivalent abnormalities were gastritis (40\%), oesophagitis $(33 \%)$, nodularity (18\%), stricture (16\%) and food bolus obstruction (10\%).

Conclusions There is little difference in demographic characteristics between interval and incident UGI cancers, however there are distinctions in presenting symptoms and endoscopic findings. Abnormalities found at the initial endoscopy prior to diagnosis of interval cancers are common.

\section{P5 1 THE REAL WORLD EFFECTIVENESS OF NEW GENERATION SPATZ BALLOON ON WEIGHT LOSS IN SUPER-OBESE PATIENTS}

${ }^{1}$ Mohammed Khan, ${ }^{1}$ Adnan Al-Zanbagi, ${ }^{1}$ Abdulaziz Tashkandi, ${ }^{2}$ Ali Montashery, ${ }^{1}$ Abdulwahab Neyazi, ${ }^{1}$ Laeeque Ahmed, ${ }^{3}$ Ghadeer Alhazmi ${ }^{*}$, ${ }^{3}$ Ghadeer Monshi, ${ }^{1}$ Mohammed Kareem Shariff. 'Digestive and Liver Center, King Abdullah Medical City, Makkah City, Saudi Arabia; ${ }^{2}$ Specialized Surgical Department, King Abdullah Medical City, Makkah City, Saudi Arabia; ${ }^{3}$ Collage of Medicine, Umm Al-Quraa University, Makkah City, Saudi Arabia

\subsection{6/gutjnl-2020-bsgcampus.80}

Introduction Pre-operative weight loss of at least $10 \%$ of excess body weight has shown to improve both intra and post operative outcomes especially in super-obese patients. Spatz Adjustable Balloon System ${ }^{\circledR}$ (Spatz FGIA, Inc., NY, USA) (SAB3) is the only Intragastric balloon whose volume can be 